\title{
Descripción de talos espermatangiales y combinación de fases en Polysiphonia confusa (Rhodomelaceae, Rhodophycota)
}

\author{
Description of spermatangial thalli and mixed-phase in Polysiphonia confusa (Rhodomelaceae, \\ Rhodophycota)
}

\author{
Raúl Aguilar-Rosas ${ }^{1 *}$, Luis E. Aguilar-Rosas² y Francisco F. Pedroche ${ }^{3}$ \\ ${ }^{1}$ Facultad de Ciencias Marinas. Universidad Autónoma de Baja California; Km 107 carretera Tijuana-Ensenada, 22830, Ensenada, Baja California, \\ México. \\ ${ }_{2}^{2}$ Instituto de Investigaciones Oceanológicas. Universidad Autónoma de Baja California; Km 107 carretera Tijuana-Ensenada, 22830, Ensenada, Baja \\ California, México. \\ ${ }^{3}$ Departamento de Hidrobiología, UAM-Iztapalapa, Apartado postal 55-535, México, D.F. 09340, Mexico y University Herbarium, University of Califor- \\ nia 1001, VLSB 2465, Berkeley, CA 94720-2465, USA \\ *Correspondencia: raguilar@uabc.mx
}

Resumen. Se describen por primera vez los talos espermatangiales, estadio que permite comparar y asociar a Polysiphonia confusa Hollenberg (Rhodomelaceae, Rhodophycota) con otras especies del género Polysiphonia. También se registra la presencia simultanea de las fases tetrasporangial y carposporangial en $P$. confusa. Se estudió el hábito, la morfología vegetativa y reproductora de $P$. confusa recolectada en la zona intermareal rocosa de Playa Saldamando, Baja California, México. El tamaño pequeño de esta especie puede justificar el número reducido de citas de este taxon para la costa del Pacífico de México.

Palabras clave: Baja California, México, algas rojas, fases reproductoras, Ceramiales.

\begin{abstract}
The spermatangial phase and the mixed phases (tetrasporangia and cystocarps) on the same thallus in Polysiphonia confusa Hollenberg (Rhodomelaceae, Rhodophycota) are described for the first time. Spermatangial thalli are important to characterize and compare this species with others from the genus Polysiphonia. Habit, vegetative, and reproductive development have been examined in plants of this species from the intertidal zone of Saldamando Beach, Baja California, México. Because of its small size, this species has been overlooked in the Mexican Pacific coast resulting in a small number of records for this taxon.
\end{abstract}

Key words: Baja California, México, red algae, reproductive phases, Ceramiales.

\section{Introducción}

El género Polysiphonia Greville, comprende alrededor de 150 especies descritas de algas rojas que taxonómicamente se distinguen por diversos caracteres, entre los que se incluyen atributos morfológicos, como el número de células pericentrales por segmento axial, el origen de los rizoides, las ramas y cuerpos espermatangiales y la frecuencia de tricoblastos. Entre las propiedades reproductoras se encuentran la disposición de los tetrasporangios y algunas características del procarpo; en concreto, el número de células que constituyen la rama carpogonial (Kim y Lee, 1999; Guimarães et al., 2004).

El epíteto de Polysiphonia confusa data de 1961 (Hollenberg, 1961), cuando Hollenberg lo estableció como un nombre nuevo (nov. nom.) para la especie $P$. inscospicua

Recibido: 17 octubre 2005; aceptado: 01 marzo 2006
Hollenberg (Hollenberg, 1944, p. 470) la cual representaba un homónimo tardío de P. inscospicua Reinsch (Reinsch, 1888, p. 146). El material original de $P$. confusa fue recolectado en rocas de la zona intermareal media cerca de Corona del Mar, Orange County, California, EUA y resultó estar formado sólo por plantas tetraspóricas sin la presencia de talo sexual. La distribución registrada abarcó sólo el sur de California y la costa occidental de Baja California (Hollenberg, 1961). Posteriormente, esta especie fue citada por Dawson et al. (1964) para el Perú. Al realizar un estudio monográfico de las especies de Polysiphonia en la parte norte del Golfo de California, México, Hollenberg y Norris (1977) citan la presencia de $P$. confusa en la costa de Sonora y por primera vez describen las plantas con cistocarpos. Hasta entonces, las plantas espermatangiales no se habían encontrado.

Distintos estudios realizados en representantes de la familia Rhodomelaceae a lo largo de las costas del Pacífico de México, como el litoral de Michoacán (Sentíes et al., 
1990; Dreckmann et al., 1990; Sentíes et al., 1992; Stout y Dreckmann, 1993), Guerrero (López et al., 2000; López et al., 2004) y Oaxaca (Galindo-Villegas et al., 1997; Mendoza-González y Mateo-Cid, 1998), han descrito plantas tetraspóricas y cistocárpicas de $P$. confusa y a pesar de que estos estudios incluyen las recolectas de varias especies del género Polysiphonia, no se registran plantas espermatangiales.

Los únicos registros de Polysiphonia confusa para la costa occidental de Baja California, corresponden a poblaciones tetraspóricas localizadas en Punta Descanso, Cabo Colonet, Punta Baja y Desembarcadero de Miller (Hollenberg, 1961). En el presente trabajo, se describen por primera vez las plantas espermatangiales en esta especie y la presencia simultánea de fases tetrasporangial y carpospangial (cistocarpos) en talos procedentes de Playa Saldamando, Baja California, México.

\section{Materiales y métodos}

Se realizaron campañas de recoleta de algas marinas en Playa Saldamando, Baja California, México, durante octubre del año 2004 (Fig. 1). Durante la recolecta se detectó in situ un crecimiento abundante de Polysiphonia confusa. Las muestras se etiquetaron y preservaron en una solución de formol al $4 \%$ con agua de mar. La determinación del material se basó en las descripciones de Abbott y Hollenberg (1976), Hollenberg (1961), Hollenberg y Norris (1977) y Sentíes (1995).

Para las observaciones al microscopio, se seleccionaron 80 fragmentos vegetativos o ramas fértiles del material conservado, se tiñeron con anilina azul al $1 \%$ en agua destilada y se montaron en una solución acuosa de miel Karo® al $20 \%$. Los cortes transversales de los ejes se realizaron con ayuda de un micrótomo de congelación. Las fotografías se tomaron con una cámara digital (Sony DSCS85, Tokio, Japón) en un microscopio Zeiss (Axioscop 40, Goettingen, Alemania). Las preparaciones permanentes y especímenes secos de herbario quedaron depositados en el Herbario CMMEX (Holmgren et al., 1990) con el registro 4432 (leg. R. Aguilar-Rosas 3206), de la Facultad de Ciencias Marinas, perteneciente a la Universidad Autónoma de Baja California en Ensenada, Baja California, México.

\section{Resultados}

Las plantas recolectadas de Polysiphonia confusa fueron de color rojo parduzco, constituidas por ejes postrados enmarañados, fijas al sustrato por rizoides, a partir de los cuales surgen los ejes erectos, con escasas ramificaciones laterales. La altura de los talos osciló entre $0.8-1.2 \mathrm{~cm}$, los ejes postrados con un diámetro de 130-200 $\mu \mathrm{m}$, fijos al

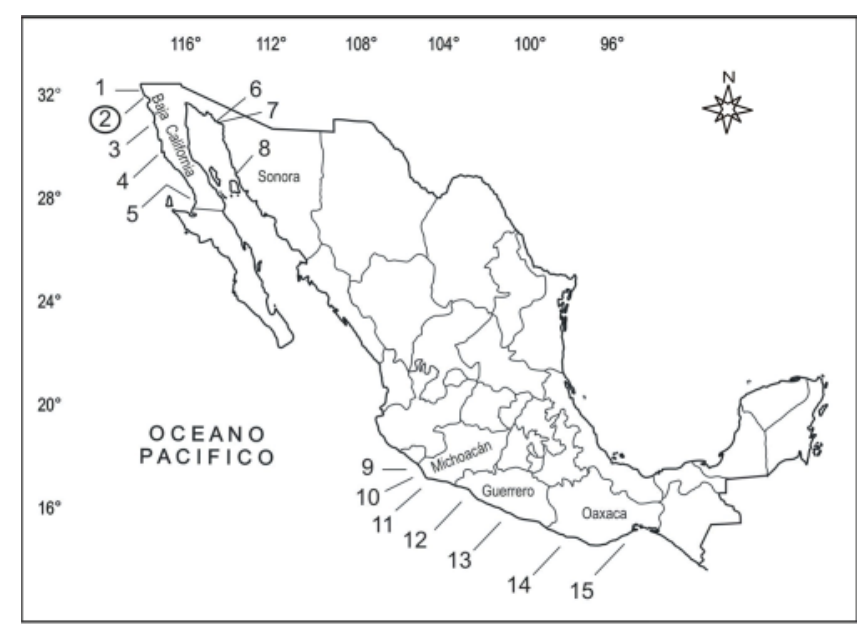

Figura 1. Localización del sitio de muestreo $(\mathrm{O})$ y distribución geográfica de Polysiphonia confusa en la costa del Pacífico de México. 1. Punta Descanso, 2. Playa Saldamando, 3. Cabo Colonet, 4. Punta Baja, 5. Desembarcadero de Miller, 6. Bahía La Cholla, 7. Puerto Peñasco, 8. Desemboque de San Ignacio, 9. Pichilinguillo, 10. Punta San Telmo, 11. Faro de Bucerias, 12. Ensenada de los Presos, 13. El Yunque, 14. Escollera del Puerto de Salina Cruz, 15. Cerro Hermoso y El Zapotal.

sustrato por medio de rizoides unicelulares que nacen de las pericentrales en conexión intercelular; éstos tienen puntas digitadas; ejes erectos de 80-140 $\mu \mathrm{m}$ de diámetro, segmentos de 1.0-2.5 más anchos que largos, con pocas ramas laterales, subdicotómicas; ramas laterales en su mayoría exógenas en asociación con los tricoblastos a intervalos irregulares y angostas de la base; 8-10 células pericentrales (Fig. 2). Los ejes erectos tienen abundantes tricoblastos con 1 o 2 dicotomías, dispuestos uno en cada segmento y alcanzando una longitud de $150-410 \mu \mathrm{m}$. Los tricoblastos son deciduos dejando cicatrices celulares conspicuas.

El material recolectado incluyó plantas tetrasporangiales, carposporangiales y espermatangiales. Los tetrasporangios son esféricos, de 60-90 $\mu \mathrm{m}$ de diámetro, dispuestos en series espiraladas en las porciones terminales de los ejes erectos (Fig. 3). Los cistocarpos son globulares de 170-280 $\mu \mathrm{m}$ de diámetro, formados lateralmente sobre las ramas (Fig. 4). Las carposporas son piriformes, de 100-140 $\mu$ m de alto y 30$40 \mu \mathrm{m}$ de diámetro. Las plantas masculinas originan ramas espermatangiales, densamente dispuestas, en los ápices de las ramas más jóvenes (Fig. 5). Los espermatangios rectos o ligeramente curvos, cilíndricos de 120-230 $\mu \mathrm{m}$ de largo y 30-55 $\mu \mathrm{m}$ de ancho se encontraron asociados a la célula inicial del tricoblasto (Fig.6). Plantas "anómalas" mostraron la presencia simultánea de tetrasporangios y cistocarpos sobre un mismo talo (Fig. 7).

Polysiphonia confusa se conoce desde el sur de California, EUA, hasta el Perú (Dawson et al., 1964; Abbott y Hollenberg, 1976). En la costa del Pacífico de México se ha registrado en 14 sitios (Fig. 1), repartidos entre los estados de Baja California, Sonora, Michoacán, Guerrero y 


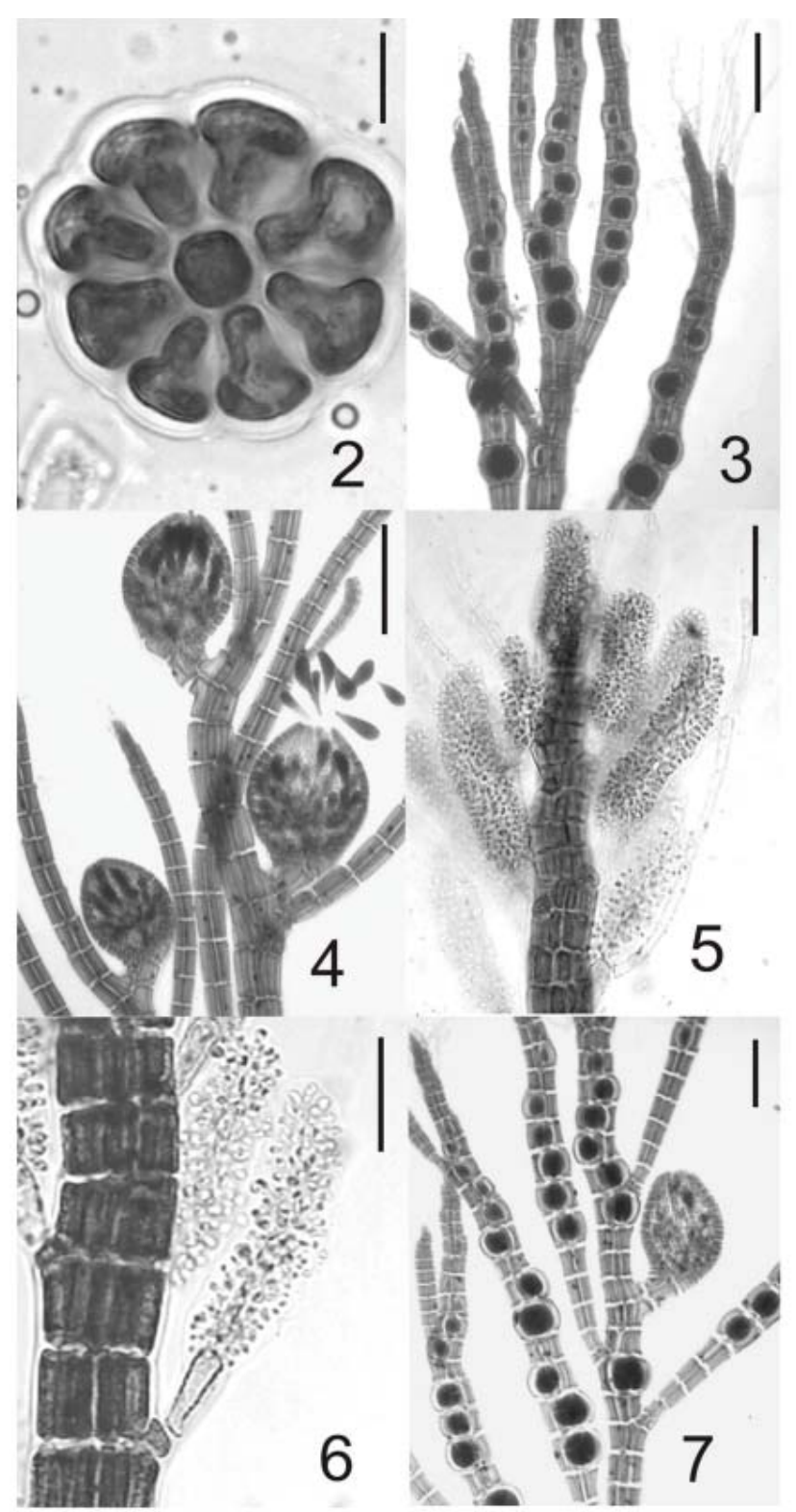

Figuras 2-7. Polysiphonia confusa (CMMEX 4432). 2, corte transversal mostrando 8 células pericentrales y una célula axial $($ escala $=25 \mu \mathrm{m}) ; 3$, aspecto general de ramas tetrasporangiales y ordenamiento en espiral de los tetrasporangios (escala $=200$ $\mu \mathrm{m}) ; 4$, cistocarpos en forma globular. Se observan carposporas recién liberadas (escala $=200 \mu \mathrm{m}) ; 5$, aspecto general de una rama espermatangial $($ escala $=200 \mu \mathrm{m}) ; 6$, espermatangio asociado a la célula inicial del tricoblasto (escala $=100 \mu \mathrm{m}) ; 7$, combinación de fases, tetrasporangios y cistocarpo sobre un mismo talo (escala = $200 \mu \mathrm{m})$.

Oaxaca (Hollenberg y Dawson, 1961; Hollenberg y Norris, 1977; Sentíes et al., 1990; Dreckmann et al., 1990; Sentíes et al., 1992; Stout y Dreckmann, 1993; Sentíes, 1995; López et al., 2000; López et al., 2004; Galindo Villegas et al., 1997; Mendoza-González y Mateo-Cid, 1998). Esta especie fue recolectada en Playa Saldamando, creciendo sobre sustrato rocoso en la zona intermareal media en ambientes expuestos al oleaje. Las pequeñas poblaciones de esta especie forman tapetes de césped; de tal manera, que habitualmente retienen cierta cantidad de arena. En el sitio de muestreo se encontraron, asociadas a la población de P. confusa, Ulva californica Wille, Endarachne binghamiae J. Agardh, Corallina vancouveriensis Yendo y Bossiella orbigniana (Decaisne) Silva.

\section{Discusión}

Los ejemplares recolectados en Baja California corresponden en general con las descripciones previas realizadas de Polysiphonia confusa, para el Pacífico de México y de California, EUA (Hollenberg, 1961; Hollenberg y Norris, 1977; Abbott y Hollenberg, 1976; Sentíes, 1995), presentándose sólo ligeras diferencias en las dimensiones de los tetrasporangios y cistocarpos.

Por ejemplo, el diámetro de los tetrasporangios (60$90 \mu \mathrm{m})$ del material examinado fue ligeramente mayor a los intervalos de 60-80 $\mu \mathrm{m}$ señalados en la descripción de especímenes para la parte norte del Pacífico de México (Baja California y Sonora) y California, EUA. Por otra parte, el diámetro de los tetrasporangios de los organismos descritos para el Pacífico tropical mexicano es entre 37 y $58 \mu \mathrm{m}$ menor. El diámetro de los cistocarpos (170-280 $\mu \mathrm{m})$ de los talos analizados es mayor en $55 \mu \mathrm{m}$ a lo registrado previamente $(225 \mu \mathrm{m})$ en la descripción de los ejemplares recolectados en la costa de Sonora (Golfo de California) por Hollenberg y Norris (1977). La diferencia de medidas registrada en los tetrasporangios, se atribuye a diferentes fases de maduración en los ejemplares, al ser recolectados.

En general, las tallas de las "plantas" y el diámetro de los ejes postrados y erectos, están dentro de los intervalos conocidos (Sentíes, 1995; López et al., 2004). La proporción largo/ancho de los segmentos de los ejes erectos de nuestros ejemplares es de 1.0-2.5, similar a lo establecido por Hollenberg (1961) y Hollenberg y Norris (1977), a diferencia de la proporción de 2.5-3.0 veces más anchos que largos registrada para el material de la costa de Michoacán en el Pacífico tropical mexicano por Sentíes et al. (1990) y Sentíes (1995).

Las plantas de Polysiphonia confusa se encontraron durante el mes de octubre (otoño), creciendo sobre sustrato rocoso en la zona intermareal media en Playa Saldamando. En otras localidades del Pacífico de México, se han citado sobre rocas o epífita de otras algas (Hollenberg y Norris, 1977; Dreckmann et al., 1990; Sentíes, 1995), desde la zona intermareal media hasta la zona submareal entre 6-12 $\mathrm{m}$ de profundidad (López et al., 2000).

Según Sentíes (1995), P. confusa puede confundirse con $P$. hendryi Gardner. Los caracteres que las diferencian son el número de células pericentrales (8-10) de $P$. confusa en contraste con las que presenta $P$. hendryi (10-12) y el nacimiento de los rizoides en conexión abierta, los cuales 
son característicos de P. hendryi (Abbott y Hollenberg, 1976; Sentíes, 1995). De la misma manera, $P$. confusa es parecida a $P$. paniculata Montagne en sus caracteres estructurales; sin embargo, $P$. paniculata mide $(5-20 \mathrm{~cm})$ y tiene mayor número de células pericentrales (10-12). Independientemente de la fase de maduración y como se ha mencionado arriba, estas tres especies se pueden distinguir por el número de células pericentrales, el tamaño de las "plantas" y el nacimiento de los rizoides.

Los talos tetrasporangiales de Polysiphonia confusa, son los más comunes en las poblaciones registradas para el Pacífico de México (Mendoza-González y Mateo-Cid, 1998), seguidos de los carposporangiales, que sólo han sido citados para Sonora (Golfo de California) y Oaxaca (Galindo-Villegas et al., 1997). El hallazgo de organismos con tetrasporangios, cistocarpos, espermatangios y la combinación de las fases tetrasporangial y carposporangial (cistocarpos) sobre un mismo talo en Baja California, nos permite ampliar el conocimiento de los caracteres morfológicos y reproductivos de $P$. confusa, de la biología reproductiva de esta especie en el Pacífico mexicano y de ciertas particularidades consideradas como anómalas por ciertos autores (Knaggs, 1969; West y Hommersand, 1981; Choi y Lee, 1996).

Cabe mencionar que para algunas especies de Polysiphonia registradas para la costa del Pacífico de México, tanto los cistocarpos como los espermatangios no se conocen en la naturaleza y su importancia como elementos de distinción taxonómica es determinante para ubicar adecuadamente a ciertos taxa (Sentíes , 1992). Por ejemplo, en Polysiphonia atlantica Kapraun et J. Norris, P. morrowii Harvey, $P$. subtilissima Montagne y P. stricta (Dillwyn) Greville, entre otras, las ramas espermatangiales se originan al iniciar las ramas laterales, desplazando a los tricoblastos (Yoon, 1986; Kim et al., 1994; Kim et al., 2000), mientras que en los talos de $P$. confusa, tienen su origen a partir de una rama del tricoblasto, asociado a la célula inicial del mismo.

La presencia simultánea de fases mezcladas ha sido registrada en miembros de las familias Ceramiaceae, Gracilariaceae y Rhodomelaceae (Rhodophycota) (Knaggs, 1969; West y Hommersand, 1981; Choi y Lee, 1996). Esta situación considerada anómala, con dos fases reproductivas (tetrasporangios, carpospororangios y espermatangios) sobre una misma planta, se ha reconocido en diversas especies de Polysiphonia en cultivo o recolectadas del ambiente natural (Edelstein y McLachlan, 1967; Lawson y Russell, 1967; Rueness, 1973; Kapraun, 1977, 1979; Cheung , 1984; Lee y Lee, 1991). Posiblemente, la mezcla de fases representa una expresión de la estructura reproductiva de los genes, debido a la mutación de los alelos que determinan el sexo (Choi y Lee, 1996).

Sin embargo, estudios citogenéticos o moleculares podrían elucidar si talos con composición genética 2n (talos tetrasporangiales), que normalmente sufren meiosis para dar lugar a esporas (tetrasporas) con la mitad del número cromosómico y las cuales germinarán para originar por su parte talos gametofitos (n) dioicos, pudieran producir también por meiosis, células que formen ramas carpogoniales, resultando en una fase "parásita" que permite la recombinación y la generación de carposporas $2 n$ en plantas ya de origen $2 n$, o bien, que estos talos tetrasporangiales representen una fuente de poliploidia o de apomixis entre las algas.

La combinación de fases tetrasporangial (2n) y carposporangial (n) sobre un mismo t talo que se encontró en $P$. confusa, es el segundo caso de esta anomalía reproductiva registrado para Baja California; ya que Aguilar-Rosas y Aguilar-Rosas (1984) informaron de un caso similar en plantas de Polysiphonia paniculata, recolectadas en San José, siendo éste el único caso de fases mezcladas en el género Polysiphonia, previamente conocido para la costa del Pacífico de México.

Si nos basamos en las pocas citas que han sido publicadas para el Pacífico de México, $P$. confusa puede considerarse un elemento relativamente raro de la ficoflora marina. El limitado número de referencias puede estar relacionado con la dificultad para localizar esta especie sobre el terreno como consecuencia del tamaño pequeño de las plantas, que en general va de 8 a $15 \mathrm{~mm}$ (30 mm). Nuevos estudios sobre las algas que intervienen en las comunidades cespitosas del intermareal, posiblemente incrementarán el número de citas de este taxon para Baja California, ampliarán su distribución regional (actualmente sólo se conoce de Punta Descanso, Punta Colonet, Punta Baja y Desembarcadero de Miller), y completarán los conocimientos actuales sobre su variación estacional y reproductiva.

Por otra parte, se requieren estudios taxonómicos más detallados sobre las especies de Polysiphonia, presentes en la costa del Pacífico de México, ya que recientemente se han propuesto combinaciones nuevas, al ser transferidas algunas especies de este género a Neosiphonia Kim y Lee (Kim, 2003; Guimaraes et al., 2004), utilizando criterios morfológicos. Neosiphonia se caracteriza por poseer ramas laterales y tricoblastos producidos en segmentos sucesivos, por la ausencia de un sistema basal postrado, por los rizoides originados por división de la célula pericentral y el arreglo en espiral de los tetrasporangios.

\section{Agradecimientos}

A la Facultad de Ciencias Marinas y al Instituto de Investigaciones Oceanológicas de la Universidad Autónoma de Baja California, por el apoyo financiero a través de la $10^{\mathrm{a}}$ Convocatoria de Investigación UABC No 571, para la realización del presente trabajo. Al Dr. A. Sentíes Granados por la revisión crítica del manuscrito. Por la asistencia en las tomas fotográficas, a Adán Castillo García. 


\section{Literatura citada}

Abbott, I. A. y G. J. Hollenberg. 1976. Marine algae of California. Stanford University Press, California. 827 p.

Aguilar-Rosas, R. y M. A. Aguilar-Rosas. 1984. Presencia de las fases carposporofita y tetrasporofita sobre el mismo talo en Polysiphonia paniculata Mont. Ciencias Marinas 10: 181-183.

Choi, H.-G y I. K. Lee. 1996. Mixed-phase reproduction in Dasysyphonia chejuensis (Rhodophyta) from Korea. Phycologia 35: 9-18.

Cheung, K. W., K. Y. Lee y I.J. Hudgkiss. 1984. The occurrence of tetrasporangia and cystocarps on the same thalli in Polysiphonia harlandii (Rhodophyta: Ceramiales). Botánica Marina 27: 571-572.

Dawson, E. Y., C. Acleto y N. Foldvik, 1964. The seaweeds of Peru. Nova Hedwigia 13: 1-111.

Dreckmann, E. K., F. F. Pedroche y A. Sentíes. 1990. Lista florística de las algas marinas bentónicas de la costa norte de Michoacán, México. Boletín de la Sociedad Botánica de México 50: 19-42.

Edelstein, T. y J. McLachlan. 1967. Cystocarps and tetrasporangia on the same thallus in Membranoptera alata and Polysiphonia urceolata. British Phycological Bulletin 3: 185-187.

Galindo-Villegas, J., J. A. Gamboa y K. M. Drekmann. 1997. Estudio de las macroalgas marinas del puerto de Salina Cruz en el Golfo de Tehuantepec, Pacífico tropical mexicano. Polibotánica 4: 1-9.

Guimarães, S. M. P. B., Fujii, M. T. Pupo D. y N. S. Yokoya. 2004. Reavaliação das características morfológicas a suas implicações taxonômicas no gênero Polysiphonia sensu lato (Ceramiales, Rhodophyta) do litorales dos Estados de São Paulo e Espiritu Santo, Brasil. Revista Brasileira de Botânica 27: 163-183.

Hollenberg, G. J. 1944. An account of the species of Polysiphonia on the Pacific coast of North America. II. Polysiphonia. American Journal of Botany 31: 474-483.

Hollenberg. 1961. Marine red algae of Pacific Mexico. Part 5. The genus Polysiphonia. Pacific Naturalist 2: 345-375.

Hollenberg, G. J. y J. N. Norris. 1977. The red alga Polysiphonia (Rhodomelaceae) in the Northern Gulf of California. Smithsonian Contributions Marine Sciences 1: 1-21.

Holmgren, P. K., N. H. Holmgren y I. C. Barnett. 1990. Index Herbariorum, Pt 1: The herbaria of the world, $8^{\text {th }}$ ed. New York Botanical Garden, New York, Bronx. x + 693 p. [Regnum Vegetabile, vol. 20].

Kapraun, D. F. 1977. Asexual propagulos in the life history of Polysiphonia ferulacea (Rhodophyta, Ceramiales). Phycologia 16: 417-426.

Kapraun, D. F. 1979. Comparative studies of Polysiphonia urceolata from three North Atlantic sites. Norway Journal Botany 26: 269-276.
Knaggs, F. W. 1969. A review of florideophycidean life histories and the culture techniques employed in their investigationn. Nova Hedwigia 18: 293-330.

Kim, M. S. 2003. Taxonomic reassessment of the genus Polysiphonia (Rhodomelaceae, Rhodophyta): Morphology and reproduction of Neosiphonia decumbens and N. harlandii. Algae 18: 107-119.

Kim, M. S. y I. K. Lee, 1999. Neosiphonia flavimarina gen. et sp. nov. with a taxonomic reassessment of the genus Polysiphonia (Rhodomelaceae, Rhodophyta). Phycological Research 47: 271-281.

Kim, M. S., I. K. Lee y S. M. Boo. 1994. Morphological studies of the red alga Polysiphonia morrowii Harvey on the Korean Coast. Korean Journal of Phycology 9:185192.

Kim, M. S., C. A. Maggs, L. McIvor y M. C. Guiry. 2000. Reapraisal of the type species of Polysiphonia (Rhodomelaceae, Rhodophyta). European Journal of Phycology 35:83-92.

Lawson, R. P. y G. Russell. 1967. Simultaneous ocurrence of carposporophytes and tetrasporangia in Polysiphonia urceolata. British Phycological Bulletin 3: 249-250.

Lee, W. J. y I. K. Lee. 1991. Mixed phases reproduction of Polysiphonia morrowii Harvey (Rhodomelaceae, Rhodophyta) in cultura. Japanese Journal of Phycology 39: 115-121.

López, N., D. Rodríguez y C. Candelaria. 2004. Intraspecific morphological variation in turf-forming algal species. Universidad y Ciencia 1: 7-15.

López, N., D. Rodríguez, C. Candelaria y J. GonzálezGonzález. 2000. Subtidal macroalgal communities of Acapulco and Zihuatanejo, Guerrero, Mexico. In Aquatic ecosystem of Mexico: status and scope, M. Munawar, S. G. Lawrence, I. F. Munawar y D. F. Malley (eds.). Ecovision World Monograph Series. Backhuys, Leiden, The Netherlands, p. 335-351.

Mendoza González, C. y L. E. Mateo-Cid. 1998. Avance de un estudio sobre las macroalgas de Guerrero y Oaxaca, México. Ciencia y Mar 1: 15-29.

Reinsch, P. F. 1888. Species et genera nova algarum ex insula Georgia Australi. Berichte der Deutschen Botanischen Gesellschaft 6: 144-156.

Rueness, J. 1973. Speciation in Polysiphonia (Rhodophyceae, Ceramiales) in view of hybridization experiments of $P$. hemisphaerica and P. boldii. Phycologia 12: 107-109.

Sentíes, G. A. 1995. El género Polysiphonia (Ceramiales: Rhodomelaceae) en el Pacífico tropical mexicano. Revista de Biología Tropical 43: 39-54.

Sentíes, G. A., M. Cordeiro-Marino y F. F. Pedroche. 1992. Taxonomía de Polysiphonia Grev.enel Pacíficomexicano. Evaluación de las características morfo-anatómicas. In Anales del II Congreso Latinoamericano de Ficología Marina. Revista de la Facultad de Oceanografía, Pesquería y Ciencias Alimentarias 2: 193-209.

Sentíes, G. A., F. F. Pedroche y K. M. Dreckmann. 1990. La 
familia Rhodomelaceae (Ceramiales, Rhodophyta) en la costa del estado de Michoacán. Boletín de la Sociedad Botánica de México 50: 89-120.

Stout, I.y K. M. Dreckmann. 1993. Macroalgas marinas de Faro de Bucerías, Michoacán, México. Anales Instituto Biología. Serie Botánica 64: 1-23.
Yoon, H.Y. 1986. A taxonomic study of genus Polysiphonia (Rhodophyta). Korean Journal of Phycology 1:3-86.

West, J. A. y M. H. Hommersand. 1981. Rhodophyta: life histories. In The biology of seaweeds. Lobban, C. S. y M. J. Wynne (eds.) Botanical Monographs 17. Blackwell, Oxford. Pp. 133-193. 